\title{
Muon and hydrogen states in II-VI semiconductor compounds. A $\mu$ SR study
}

\author{
A. Weidinger ${ }^{1}$, H. V. Alberto ${ }^{*}, 2$, J. M. Gil ${ }^{2}$, R. C. Vilão ${ }^{2}$, J. Piroto Duarte ${ }^{2}$, \\ and N. Ayres de Campos ${ }^{2}$ \\ ${ }^{1}$ Hahn-Meitner Institut Berlin, Glienicker Strasse, 100, 14109 Berlin, Germany \\ ${ }^{2}$ Physics Department, University of Coimbra, 3004-516 Coimbra, Portugal
}

Received 24 July 2002, revised 4 August 2002, accepted 4 August 2002

Published online 30 January 2003

PACS 61.72.Vv, 71.55.Gs, 76.75.+i

\begin{abstract}
Additional hydrogen in II-VI semiconductors may occur in two different configurations, either bound to the anion in a position along the bond direction, or at an open interstitial site surrounded by four cations. The electronic character of these states is very different: the bound hydrogen forms a donor with an extended electronic wave function, whereas the interstitial hydrogen is acceptor-like and has an almost freeatom-like electron distribution. In this paper, we show in which systems the donor and in which the acceptor was found to form the ground state. Some of the structural and electronic properties of these states are also given. The information presented is derived from $\mu \mathrm{SR}$ experiments.
\end{abstract}

Introduction Hydrogen as a dopant in $\mathrm{ZnO}$ was studied extensively in the fifties (e.g. [1, 2]). From transport measurements, a donor level energy of $51 \mathrm{meV}$ was derived [2].

The first spectroscopic information on a hydrogen-like shallow donor state in a II-VI compound was reported by Gil et al. [3] for muonium in CdS. Almost simultaneously a theoretical paper predicting a shallow donor state in $\mathrm{ZnO}$ [4] stimulated a successful experimental search for this state by $\mu$ SR [5]. Recently, an ESR/ENDOR study on hydrogen in $\mathrm{ZnO}$ was published [6] and a theoretical paper on hydrogen in various oxides [7] came out.

It is generally agreed that the donor state corresponds to hydrogen bound to the anion $(\mathrm{O}, \mathrm{S}, \mathrm{Se}, \mathrm{Te})$ of the II-VI compound, with a position either near the bond centre or near the antibonding site. A competing configuration is also observed where interstitial hydrogen is surrounded by four positively charged cations. This latter configuration gives rise to an acceptor state. The question is: Which of the two states is lower in energy and forms the ground state for a given system? $\mu \mathrm{SR}$ data show that the donor state is formed in $\mathrm{CdS}, \mathrm{CdSe}, \mathrm{ZnO}$, and $\mathrm{HgO}$, whereas the acceptor state is found in $\mathrm{ZnS}$ and $\mathrm{ZnSe}$. In CdTe both states are formed simultaneously. Other systems have not been checked yet, or no conclusive results were reported. The experimental results of $\mu \mathrm{SR}$ measurements are presented here.

\section{Model}

The donor state $\mathbf{H}^{+/ 0}$ or $\mathrm{Mu}^{+/ 0}$ In this configuration, the proton or muon is bound to the anion $(\mathrm{O}, \mathrm{S}$, $\mathrm{Se}, \mathrm{Te}$ ) and occupies a position near the bond-centre or near the antibonding site. In general, the electron is weakly bound to the positive charge and the binding energy corresponds to that of a shallow donor in these materials. The shallowness of the donor is not a necessary property of this configuration and we

\footnotetext{
* Corresponding author: e-mail: lena@ci.uc.pt, Fax: (+351) 239829158
} 


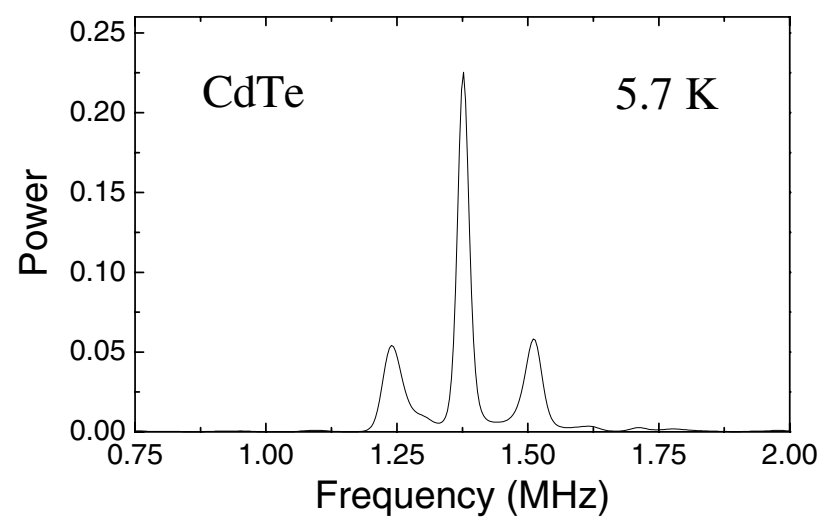

Fig. 1 Fourier transform of the $\mu$ SR spectrum for CdTe at $10 \mathrm{mT}$. The middle line corresponds to the diamagnetic fraction whereas the two lines below and above it are due to the paramagnetic donor state.

include also systems like $\mathrm{HgO}$ where a medium-deep state was found [8]. The hyperfine interactions are weak and are in general anisotropic as expected from the configuration of the state.

The acceptor state: $\mathbf{H}^{\mathbf{0} /-}$ or $\mathbf{M u}^{\mathbf{0} /-}$ The acceptor state is associated with a proton or muon at an interstitial site surrounded by the metal cations. In this interstitial position, the electron is quite strongly bound to the positive charge, almost like in a free atom.

Acceptor/donor level ordering In the "normal" ordering, the acceptor level (0/-) is energetically below the donor level (+/0) and the acceptor forms the ground state. In this case normal hydrogen or muonium with an isotropic and strong hyperfine interaction will be formed. In the "anomalous" ordering case, the donor forms the ground state, and $\mu$ SR and ENDOR results show the typical feature of weakly hyperfine splitted lines as exposed in Fig. 1.

Ground state configuration Under most Fermi level conditions, the configuration with the lower conversion level energy forms the ground state. In one exceptional situation there is a "site conversion": if the acceptor is below the donor and if the Fermi energy is below the acceptor by more than the energy difference of the conversion levels, then $\mathrm{H}^{+}$or $\mathrm{Mu}^{+}$forms the ground state. This occurs only if the acceptor is below, but not very much below the donor.

Results and discussion The donor state was clearly identified by the paramagnetic hyperfine splitting in $\mu \mathrm{SR}$ experiments for the compounds $\mathrm{CdS}, \mathrm{CdSe}, \mathrm{HgO}, \mathrm{ZnO}$ and $\mathrm{CdTe}[3,5,8,9]$. The amplitudes of the corresponding lines illustrate that the formation probability of this state is almost $100 \%$ for the first four compounds, whereas in CdTe only about $55 \%$ are associated with this signal. In CdTe, decoupling experiments show that a major fraction of the remaining muons have an interaction typical to normal muonium, i.e., $A=2400 \mathrm{MHz}$. Consequently there is also a formation of the acceptor state as described

Table 1 Formation probabilities (\%) of the different paramagnetic states in $\mathrm{Cd}$ and $\mathrm{Zn}$ chalcogenides and in the oxides $\mathrm{HgO}$ and $\mathrm{ZnO}$, observed at low temperature. The percentage was derived either directly from the amplitudes of the corresponding paramagnetic states, or indirectly by a decoupling experiment in longitudinal field.

\begin{tabular}{llllllll}
\hline & $\mathrm{CdS}$ & $\mathrm{CdSe}$ & $\mathrm{CdTe}$ & $\mathrm{HgO}$ & $\mathrm{ZnO}$ & $\mathrm{ZnS}$ & $\mathrm{ZnSe}$ \\
\hline donor & 90 & 90 & 55 & 100 & 93 & - & - \\
acceptor & & & 35 & & & 80 & 70 \\
unidentified & 10 & 10 & 10 & & 7 & 20 & 30 \\
\hline
\end{tabular}




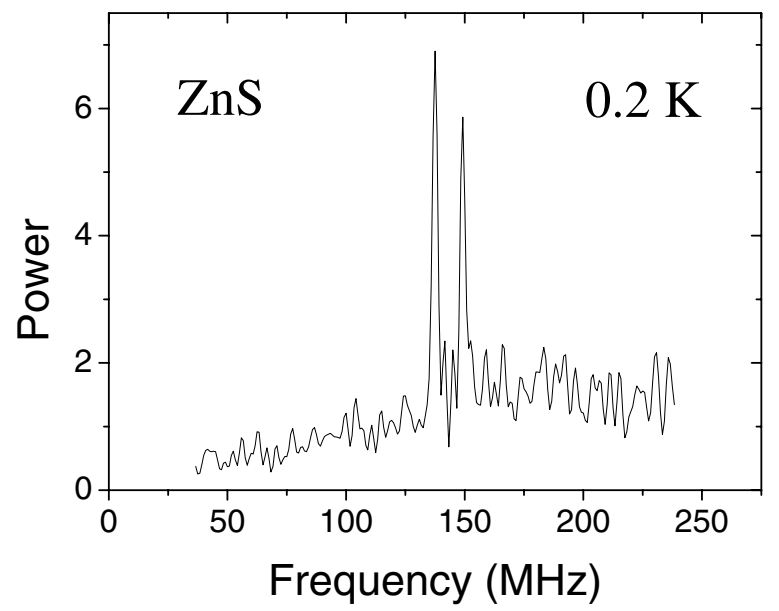

Fig. 2 Fourier transform of the $\mu$ SR spectrum for $\mathrm{ZnS}$ at $10 \mathrm{mT}$. Note the much larger frequency values than in Fig. 1. The two lines at $137 \mathrm{MHz}$ and $149 \mathrm{MHz}$, respectively, are the signature of normal muonium.

above. In all three $\mathrm{Cd}$ compounds considered here a small fraction of non-clearly identified muons has been observed. The respective percentages are shown in Table 1 .

In the $\mathrm{ZnS}$ and $\mathrm{ZnSe}$ compounds, no sign of the shallow donor state was found, but instead the normal muonium signature, i.e., the splitting in two lines at large frequencies in a transverse field of $100 \mathrm{G}$ was observed [9] (see Fig. 2). The amplitude of these oscillations is rather weak and corresponds to only $(10-30) \%$ of all muons. However, in a decoupling experiment, it could be shown that the normal muonium is formed with a rather large fraction on the order of $(70-80) \%$ [9]. The difference between the large total fraction of normal muonium formation and its observation in the transverse-field is attributed to a dephasing effect due to late formation of this state. In all cases, some unidentified fraction has been assigned which is due either to strongly relaxing signals or missing fractions. These parts may well correspond to the majority configurations in the different compounds, but a clear assignment is not possible.

As can be seen from Table 1, in $\mathrm{CdS}, \mathrm{CdSe}, \mathrm{ZnO}$ and $\mathrm{HgO}$, the formation of the donor state is clearly favoured whereas in $\mathrm{ZnS}$ and $\mathrm{ZnSe}$, the acceptor state dominates. CdTe seems to be intermediate, showing the formation of both states. The selective formation of one or the other state is considered as an indication that the corresponding state, i.e. $\mathrm{Mu}^{+/ 0}$ or $\mathrm{Mu}^{0 /-}$, is lower in energy. The fact that both centres are formed in CdTe suggests that the two states have approximately equal stability.

A direct comparison of the muonium and hydrogen state is possible for $\mathrm{ZnO}$. The ENDOR results [6] are consistent with the $\mu \mathrm{SR}$ data [5] concerning the fact that a shallow state is observed and the level energies are comparable. A rather large and not well-understood discrepancy occurs in the hyperfine values. In both cases they are very weak, as expected for a shallow donor with an extended electron wave function, but the hydrogen value is considerably larger than expected from the scaling of the muon value. A possible explanation of this discrepancy might be that the hyperfine value depends strongly on the bond length and bond angle and these might be different in the two cases. But we have no concrete justification for this assumption.

Conclusions We have discussed the two possible states of muonium (and by analogy of hydrogen) in the II-VI compounds, that is to say the donor state $\mathrm{Mu}^{+/ 0}$ and the acceptor state $\mathrm{Mu}^{0 /-}$. These states are associated with a muon bound to the anion and a fairly unbound state at an interstitial position surrounded by the cations, respectively. The formation probabilities observed at low temperatures indicate that a donor state lies lower in energy than the acceptor state in $\mathrm{CdS}, \mathrm{CdSe}, \mathrm{ZnO}$ and $\mathrm{HgO}$ whereas it is the opposite in $\mathrm{ZnS}$ and $\mathrm{ZnSe}$. In CdTe both states are formed, indicating that the level energy of the two states is similar. The different ordering of the conversion levels in $\mathrm{ZnSe}$ compared to $\mathrm{ZnO}$ was recently predicted by Van de Walle [10]. 
Acknowledgements The help of the $\mu$ SR groups at PSI and ISIS for supporting experiments is gratefully acknowledged. This work was partially supported by FCT/POCTI grant 35334/FIS/2000 (Portugal).

\section{References}

[1] E. Mollwo, Z. Phys. 138, 478 (1954).

[2] A. R. Hutson, Phys. Rev. 108, 222 (1957).

[3] J. M. Gil et al., Phys. Rev. Lett. 83, 5294 (1999).

[4] C. G. Van de Walle, Phys. Rev. Lett. 85, 1012 (2000).

[5] S. F. J. Cox et al., Phys. Rev. Lett. 86, 2601 (2001).

[6] D. M. Hofmann et al., Phys. Rev. Lett. 88, 045504 (2002).

[7] Ç. Kiliç and A. Zunger, Appl. Phys. Lett. 81, 73 (2002).

[8] J. M. Gil et al., J. Phys., Condens. Matter 13, L613-L618 (2001).

[9] J. M. Gil et al., Phys. Rev. B 64, 075205 (2001).

[10] C. G. Van de Walle, phys. stat. sol. (b) 229, 221 (2002). 